\title{
Sex Difference and Steroid Modulation of Pheromone-Induced Immediate Early Genes in the Two Zones of the Mouse Accessory Olfactory System
}

\author{
Heather A. Halem, ${ }^{1}$ Michael J. Baum, ${ }^{1}$ and James A. Cherry ${ }^{2}$ \\ Departments of ${ }^{1}$ Biology and ${ }^{2} P$ sychology, Boston University, Boston, Massachusetts 02215
}

Two anatomically and neurochemically distinct zones within the vomeronasal organ (VNO) and accessory olfactory bulb (AOB) have been identified that are responsible for the detection of pheromones. Using markers to distinguish between apical and basal neurons of the VNO neuroepithelium and rostral versus caudal $A O B$ glomeruli, we examined immediate early gene immunoreactivity (IEG-IR) in gonadectomized, steroid-treated mice in response to pheromones of male and female conspecifics. After exposure of estradiol-treated females to soiled male bedding, more VNO neurons in the basal than the apical layer exhibited IEG-IR compared with VNO neurons of estradiol-treated males. Conversely, whereas soiled female bedding failed to induce IEG-IR in VNO neurons of estradiol- treated males or females, both apical and basal neurons were activated in testosterone-treated males. Male and female pheromones also activated mitral and granule cells in the AOBs of all subjects, but responses to different pheromones were distributed across the boundary of the rostral and caudal regions. These data show that differences in the response of males and females to the same pheromonal stimulus are found in the sensory neurons of the VNO. We propose that centrifugal, noradrenergic inputs to VNO neurons, which may differ in the two sexes and respond differently to adult sex steroids, modulate sensitivity to pheromonal stimulation.

Key words: vomeronasal organ; accessory olfactory bulb; steroid hormone; pheromone; sex differences; Fos; Egr-1
Many pheromones are detected by an anatomically subdivided pathway from the vomeronasal organ (VNO) to the accessory olfactory bulb (AOB) (Jia and Halpern, 1996; Belluscio et al., 1999; Rodriguez et al., 1999). In the VNO, receptor neurons located in the apical neuroepithelium project exclusively to glomeruli in the rostral portion of the accessory olfactory bulb (AOB), whereas basal neurons send axons to caudal AOB glomeruli (Jia and Halpern, 1996; Belluscio et al., 1999; Rodriguez et al., 1999). These neurons also differ in their expression of GTPbinding (G) protein subunits (Jia and Halpern, 1996) and receptor proteins that may bind pheromones; the V1R receptors occur in $\mathrm{G} \alpha_{\mathrm{i} 2}$-expressing apical neurons (Dulac and Axel, 1995), and $\mathrm{V} 2 \mathrm{R}$ receptors are localized in $\mathrm{G} \alpha_{\mathrm{o}}$-expressing basal neurons (Herrada and Dulac, 1997; Matsunami and Buck, 1997; Ryba and Tirindelli, 1997).

A model to explain sensory transduction in the accessory olfactory system must consider not only the role of each neurochemically distinct pathway but also must account for observations that behavioral responses to the same pheromones differ depending on the sex and endocrine status of the respondent. For example, in male mice, exposure to female pheromones facilitates luteinizing hormone secretion (Johnston and Bronson, 1982; Coquelin et al., 1984) and stimulates ultrasonic vocalizations (Nyby et al., 1979), whereas male pheromones elicit intermale aggressive

Received Oct. 30, 2000; revised Jan. 5, 2001; accepted Jan. 19, 2001.

This research was supported by National Institutes of Health Grants MH59200 (M.J.B.) and DC03019 (J.A.C.). H.A.H. received support from T32 HD07387 to Boston University. We thank Diana Chen for technical assistance and Drs. Gerard Evan and David Hancock for generously providing the DCH-1 Fos antisera used in this study.

Correspondence should be addressed to Dr. James A. Cherry, Department of Psychology, 64 Cummington Street, Boston University, Boston, MA 02215. E-mail: jcherry@bu.edu.

Copyright (C) 2001 Society for Neuroscience $\quad 0270-6474 / 01 / 212474-07 \$ 15.00 / 0$ behavior (Guillot and Chapouthier, 1996). In contrast, exposure of females to female pheromones delays puberty and suppresses estrus (van der Lee and Boot, 1959), whereas male pheromones accelerate puberty (Lombardi and Vandenbergh, 1977), cause estrous cycle synchronization (Whitten, 1959), and induce a pregnancy block to recent mating (Bruce, 1959).

Perhaps differences in the behavioral responses of males and females to a particular pheromone are attributable solely to differences in central processing. Using immediate-early gene (IEG) protein expression as a marker of neuronal activation, it has been shown that chemosensory cues in soiled bedding stimulate a sexually dimorphic pattern of Fos protein immunoreactivity (IR) in central sites along the VNO projection pathway (Bakker et al., 1996; Kelliher et al., 1998; Halem et al., 1999). On the other hand, sex differences in the VNO itself may also exist that mediate sexually dimorphic responses to pheromonal cues. There is evidence that more neurons are present in the VNO of males than females (Segovia and Guillamon, 1982), and sex differences in VNO receptor expression have been reported (Herrada and Dulac, 1997).

In the following experiments, we address this issue and three questions central to the understanding of pheromone processing: do neurons in apical and basal layers of the VNO respond uniquely to different pheromones, and are these responses sexually dimorphic and modulated by sex hormones? Using markers to label specific subdivisions of the $\mathrm{VNO}$ and $\mathrm{AOB}$, we compared IEG induction in male and female mice by pheromones from both sexes. In addition, we asked whether administration of androgen or estrogen alters these responses. We show that VNO neurons in the two zones are differentially responsive to male and female pheromones, that the response is regulated by sex steroids, and that it differs in male and female subjects. 


\section{MATERIALS AND METHODS}

Subjects. Six-week-old BALB/c mice (Taconic Farms, Germantown, NY) were purchased and housed under a $12 \mathrm{hr}$ light/dark cycle (lights on at 8:00 A.M.) with food and water available ad libitum. All subjects were bilaterally gonadectomized using ketamine $(188 \mathrm{mg} / \mathrm{kg})$ and xylazine $(50$ $\mathrm{mg} / \mathrm{kg})$ anesthesia and treated daily with estradiol benzoate (EB) $(0.06$ $\mathrm{mg} / \mathrm{kg}$, s.c.) or testosterone priopionate (TP) $(3 \mathrm{mg} / \mathrm{kg}$, s.c.) for 3 weeks. Subjects were then isolated in individual cages for $4 \mathrm{~d}$ and continued to receive EB or TP. All methods were approved by the Boston University institutional use and care committee.

Exposure to pheromonal stimuli. Five stimulus females were ovariectomized and injected subcutaneously with EB $(1.25 \mathrm{mg} / \mathrm{kg}) 4$ and $2 \mathrm{~d}$ before bedding collection. On the fifth day at 4:00 P.M., females were given progesterone $(31.3 \mathrm{mg} / \mathrm{kg}$ ) and housed individually in clean plastic cages on $48 \mathrm{gm}$ of Care Fresh paper bedding (Absorption Co., Bellingham, WA). Bedding was collected $17 \mathrm{hr}$ later from all females, combined, and used the same day. Five gonadally intact stimulus males were housed individually in a clean plastic cage lined with clean bedding. Bedding was collected from 4:00 P.M. to 9:00 A.M., combined, and also used the same day. Subjects were placed individually into cages containing clean bedding, soiled male bedding, or soiled female bedding during the light phase of the day/night cycle and were killed 90 min later.

Collection of tissues. Mice were perfused with PBS, pH 7.4, followed by $15 \mathrm{ml}$ of $4 \%$ paraformaldehyde in $0.1 \mathrm{M}$ PBS. Snouts and brains were post-fixed in $4 \%$ paraformaldehyde for $4 \mathrm{hr}$ before being placed into $30 \%$ sucrose-PBS for $72 \mathrm{hr}$ at $4^{\circ} \mathrm{C}$. Snouts were frozen in OCT (Tissue-Tek, Miles Inc., Elkhart, IN) and cryosectioned coronally at $20 \mu \mathrm{m}$. Consecutive sections were mounted onto glass slides and then stored at $-80^{\circ} \mathrm{C}$. Olfactory bulbs were embedded separately in OCT and frozen on dry ice. Free-floating, $30 \mu \mathrm{m}$ sagittal sections of the right olfactory bulb were placed in $0.1 \mathrm{M}$ PBS at $4^{\circ} \mathrm{C}$ and used immediately.

Double-label immunocytochemistry. VNO and olfactory bulb sections were first processed using the single-label immunocytochemistry procedure detailed previously (Halem et al., 1999). For the VNO, a 1:1000 dilution of polyclonal antiserum raised against the $\mathrm{C}$-terminal portion of human Egr-1 (Santa Cruz Biotechnology, Santa Cruz, CA) was used, and signals were visualized with $0.025 \%$ 3,3' diaminobenzidine (DAB) with nickel intensification (Vector Laboratories, Burlington, CA). For the olfactory bulbs, free-floating sections were labeled using a 1:5000 dilution of $\mathrm{DCH}-1$, a rabbit polyclonal antiserum raised against the $\mathrm{N}$-terminal sequence of amino acids $2-17$ of rat Fos protein. Fos-IR neurons were then visualized with DAB. Neuronal nuclei with Fos-IR exhibited a purple-black reaction product (see Fig. 1).

To apply the second label, VNO sections were next incubated with a primary antibody that recognizes Type IVA phosphodiesterase (PDE4A) (Cherry and Davis, 1995), which specifically labels apical neurons in the VNO neuroepithelium (Lau and Cherry, 2000). Sections were processed as in the single-label procedure, except that Nova Red (Vector Laboratories) was used to visualize PDE4A-IR neurons, which were identified by a red reaction product in the cytoplasm, dendrites, and axons (see Fig. 1A). Fos-labeled olfactory bulb sections were exposed to a 1:1000 dilution of mouse monoclonal antibody raised against $\mathrm{G} \alpha_{\mathrm{i} 2}$ protein (Chemicon, Temecula, CA), which specifically labels glomeruli in the rostral AOB, and were visualized with VIP (Vector Laboratories).

Data analysis. Slides were coded to conceal subjects' sex, steroid treatment, and bedding stimulus. Only neurons containing a purpleblack nucleus (as opposed to light brown) were designated as containing Egr-1-IR or Fos-IR. The location of Egr-1-IR cells in every third section of the VNO neuroepithelium was recorded at $250 \times$ using a camera lucida microscope attachment. The apical-basal division of the neuroepithelium was determined by red PDE4A immunoreactivity in the apical zone (see Fig. 1). Egr-1-IR cells in the apical and basal regions of each VNO section were counted, and the mean number of Egr-1-IR cells per section was determined for apical and basal regions in each subject.

The number of Fos-IR neurons in mitral and granule cell layers of the AOB was determined separately for rostral and caudal regions. Two anatomically matched AOB sections per subject were selected, and the location of Fos-IR neurons was determined at $250 \times$ using the camera lucida. A line was drawn at the division between the $\mathrm{G} \alpha_{\mathrm{i} 2}$-IR rostral glomeruli and the nonlabeled caudal region and extended through the mitral and granule cell layers (see Fig. 1A). The mean number of Fos-IR cells in the two regions of the AOB mitral and granule cell layers was calculated for each subject. The total area of the rostral and caudal subdivisions of the mitral and granule cell layers was measured and used to compute the number of Fos-IR neurons per $0.1 \mathrm{~mm}^{2}$ for each subject (see Fig. 2).

Statistics. Because there were no IEG-IR neurons in some of the control groups, Kruskal-Wallis one-way ANOVA on ranks were first applied to establish whether the number of IEG-IR neurons counted in the VNO and AOB differed in animals exposed to soiled versus clean bedding. Separate analyses were conducted for EB-treated females (EBfemales), EB-treated males (EB-males), and TP-treated males (TPmales) for each location within the VNO (apical and basal) and the AOB (rostral and caudal). Post hoc comparisons were then performed using Mann-Whitney $U$ tests. To determine whether the mean number of IEG-IR neurons induced by male or female pheromones in the VNO and AOB depended on treatment group and neuron location, parametric two-way ANOVAs were conducted with location as a repeated measure. Separate ANOVAs were performed on IEG responses to soiled male bedding and to soiled female bedding. Student-Newman-Keuls post hoc comparisons were used to detect mean differences between groups.

Spatial diagrams. Composite diagrams for the VNO and AOB were constructed to illustrate the overall pattern of IEG responsiveness after exposure to soiled bedding. IEG-IR neurons from two anatomically similar sections in each subject were traced onto a single grid (1 square is 0.24 or $0.80 \mu \mathrm{m}^{2}$ for the $\mathrm{VNO}$ and $\mathrm{AOB}$, respectively). The mean number of IEG-IR neurons in each of the grid squares was calculated for each group of animals, and grid squares were assigned a color code on the basis of this calculation (see Fig. 3). For each composite figure, the apical-basal border in the VNO or the rostral-caudal border in the AOB was determined by overlaying camera lucida tracings from all subjects.

To determine whether overall patterns of IEG-IR in the AOB differed among groups exposed to different pheromones, two-way repeated measures ANOVAs were used to compare mean numbers of IEG-IR neurons across individual grid squares. To simplify the analysis and to focus on specific comparisons of interest, IEG responses of EB-males and EBfemales were compared in a two-way ANOVA with bedding type and sex of subject as factors. Similarly, IEG induction in EB-males and TP-males were compared in a separate two-way ANOVA with bedding type and hormone treatment as factors. For these comparisons, grid square was used as a repeated measure, and granule and mitral cell data were examined separately. Because of the large number of zero values in individual grid squares, overall patterns of IEG-IR in the VNO were not analyzed.

\section{RESULTS}

In a previous study (Halem et al., 1999), we used Fos-IR as a measure of cellular activation in the AOB and VNO after exposure to pheromones but observed only a modest induction of Fos-IR in the VNO. We have since determined that stimulation of VNO neurons by pheromones induces Egr-1 protein more robustly than the Fos antibody used previously. In the present study, we compared patterns of both Egr-1 and Fos induction in the VNO and found only quantitative differences. Therefore, detailed analyses of IEG protein induction by pheromones are presented for Egr-1 in the VNO and for Fos in the AOB.

\section{IEG Responses in the VNO}

Compared with clean bedding, exposure to soiled bedding significantly stimulated Egr-1-IR in both apical and basal neurons of all treatment groups (EB-females, EB-males, TP-males; $H>9.9$; $\mathrm{df}=2 ; p<0.05$ for all one-way ANOVAs) (Figs. 1, 2). However, as detailed below, the patterns of activation within VNO layers induced by male and female pheromones differed depending on the sex and hormone treatment of the subject.

\section{Response to male pheromones}

Exposure to soiled male bedding induced significantly more Egr1-IR neurons in the basal than the apical region of the VNO $\left(F_{(1,29)}=291.7 ; p<0.01\right)$, and overall, induction of Egr-1-IR in VNO neurons by male pheromones differed between groups $\left(F_{(2,29)}=197.3 ; p<0.01\right)$. In particular, significantly more basal than apical neurons were immunoreactive for Egr-1 in EB-males and EB-females but not TP-males $(p<0.05$; Student-Newman- 

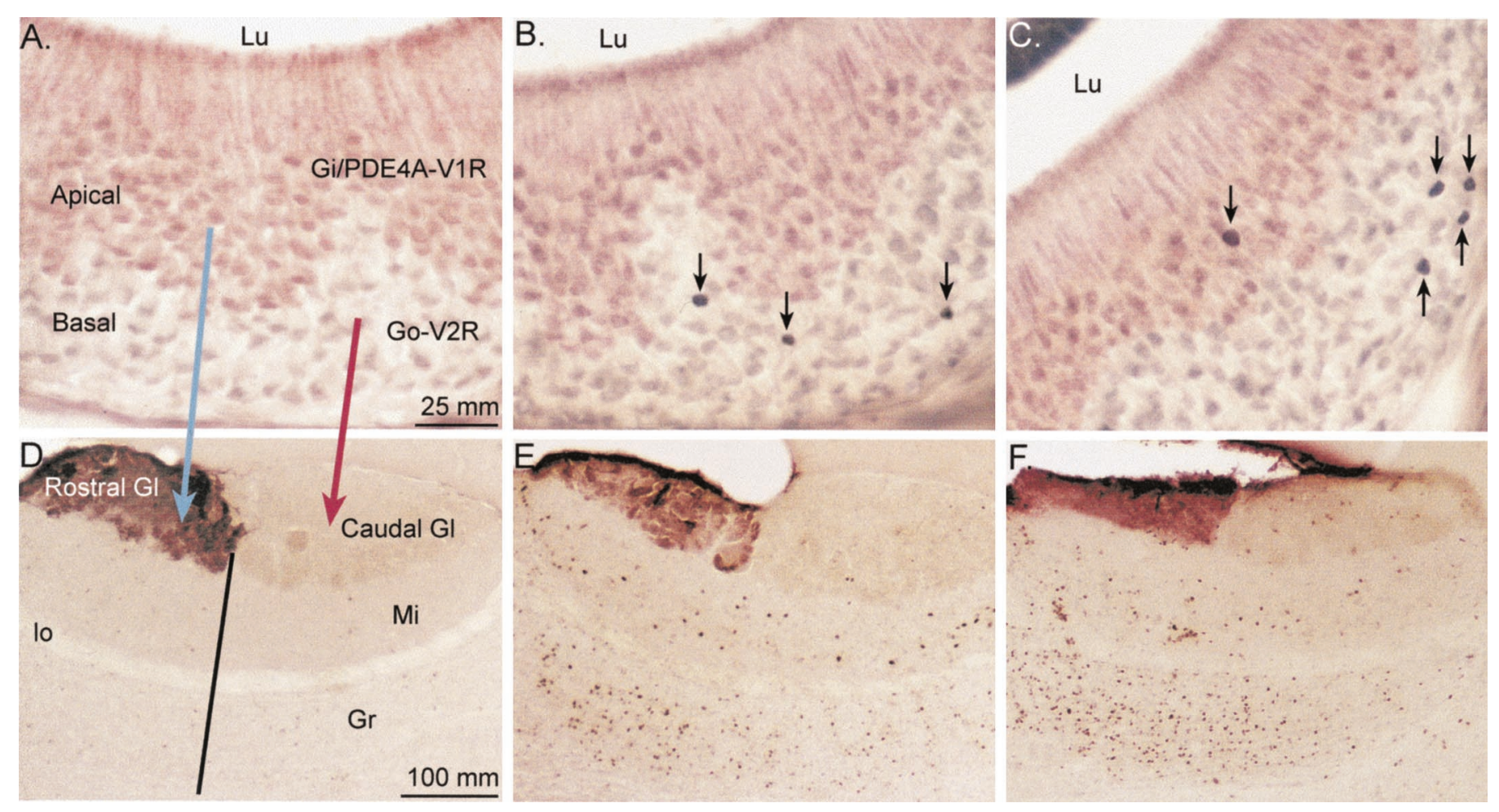

Figure 1. Representative photomicrographs are shown of Egr-1-IR neurons in the VNO $(A-C)$ and Fos-IR neurons in the AOB ( $D-F)$ of gonadectomized, steroid hormone-treated animals exposed to different sources of pheromones. $A, D$, EB-treated female exposed to clean bedding. $B$, $E$, EB-treated female exposed to soiled male bedding. $C, F$, TP-treated male exposed to soiled female bedding. Anti-PDE4A antibody was used to label the apical portion of the $\mathrm{VNO}$, and anti-G $\alpha_{\mathrm{i} 2}$ antibody was used to stain the rostral portion of the AOB glomerular layer The blue and red arrows in $A$ and $D$ depict the projection of apical and basal VNO neurons to rostral and caudal regions of the AOB, respectively. The black line in $D$ shows the division between rostral and caudal that was extended into the mitral and granule cell layers of the AOB. Black arrows in $B$ and $C$ point to Egr-1-IR neurons in the VNO neuroepithelium. $G l$, Glomerular layer; $G r$, granule cell layer; lo, lateral olfactory tract; $L u$, lumen; $M i$, mitral cell layer.

Keuls post hoc comparisons). A significant treatment $\times$ location interaction $\left(F_{(2,29)}=231.7 ; p<0.01\right)$ reflected the observation that Egr-1-IR in the female VNO was markedly greater than in either group of males, particularly in basal neurons. Fos-IR induced in VNO neurons by male pheromones in EB-males and EB-females was reduced but similar compared with Egr-1-IR (data not shown). However, no Fos-IR was observed in the VNO neuroepithelium of TP-males.

\section{Response to female pheromones}

There were no overall differences between groups in the number of Egr-1-IR neurons induced $\left(F_{(1,29)}=0.06 ; p>0.05\right)$, but exposure to female pheromones resulted in a dramatic induction of Egr-1-IR in VNO neurons of TP-males but not in EB-males or EB-females $\left(F_{(2,29)}=8.2 ; p<0.01\right)$. Post hoc tests indicated that equivalent, significant increases in neuronal Egr-1-IR occurred in the apical and basal zones of the VNO of TP-treated males. In contrast, Egr-1 (and Fos; data not shown) was induced negligibly or not at all in VNO neurons of the EB-males and EB-females.

\section{IEG Responses in the AOB}

Neurons from the VNO send their axons to glomeruli in the AOB in which synapses are made with the processes of neurons from the AOB mitral cell layer. In turn, dendrites of mitral cells form reciprocal dendrodendritic synapses with deeper-lying granule cells. We found that soiled bedding significantly stimulated Fos-IR in rostral and caudal subdivisions of mitral and granule cells in EB-male and EB-female groups $(H>5$; df $=2 ; p<0.05$ for all one-way ANOVAs) (Fig. 2). In TP-males, significantly more Fos-IR neurons were found in both the rostral and caudal mitral cell layers after exposure to soiled bedding $(H>6.9$; $\mathrm{df}=$ $2 ; p<0.05$ for both locations) (Fig. 2), as well as in the rostral $(H=6.9 ; \mathrm{df}=2 ; p<0.05)$ but not the caudal $(H=4.8 ; \mathrm{df}=2$; $p>0.05)$ granule cell layers (Table 1$)$.

\section{Response to male pheromones}

Exposure to soiled male bedding induced significantly greater numbers of Fos-IR cells in the rostral than the caudal portion of the AOB mitral cell layer $\left(F_{(1,39)}=5.4 ; p<0.05\right)$. There were, however, no overall differences in Fos-IR induction between different groups $\left(F_{(2,39)}=3.4 ; p>0.05\right)$, and post hoc comparisons did not identify any groups in which there was a significant difference between the rostral and caudal regions. In granule cells, there was a significant rostral-caudal difference in Fos-IR $\left(F_{(1,39)}=10.5 ; p<0.01\right)$, as well as differences between treatment groups in the overall number of Fos-IR neurons $\left(F_{(2,39)}=3.9 ; p<0.05\right)$. Post hoc comparisons revealed that there were significantly more Fos-IR granule cells in EB-males and EB-females than in TP-males.

\section{Response to female pheromones}

In the mitral cell layer of the AOB, there were no differences between groups in the induction of Fos-IR by soiled female bedding $\left(F_{(2,39)}=1.7 ; p>0.05\right)$; however, more Fos-IR neurons were induced overall in the rostral than the caudal AOB $\left(F_{(1,39)}=17.1 ; p<0.01\right)$. Post hoc tests indicated that significantly more Fos-IR mitral cells were present in the rostral than the 
Vomeronasal Organ

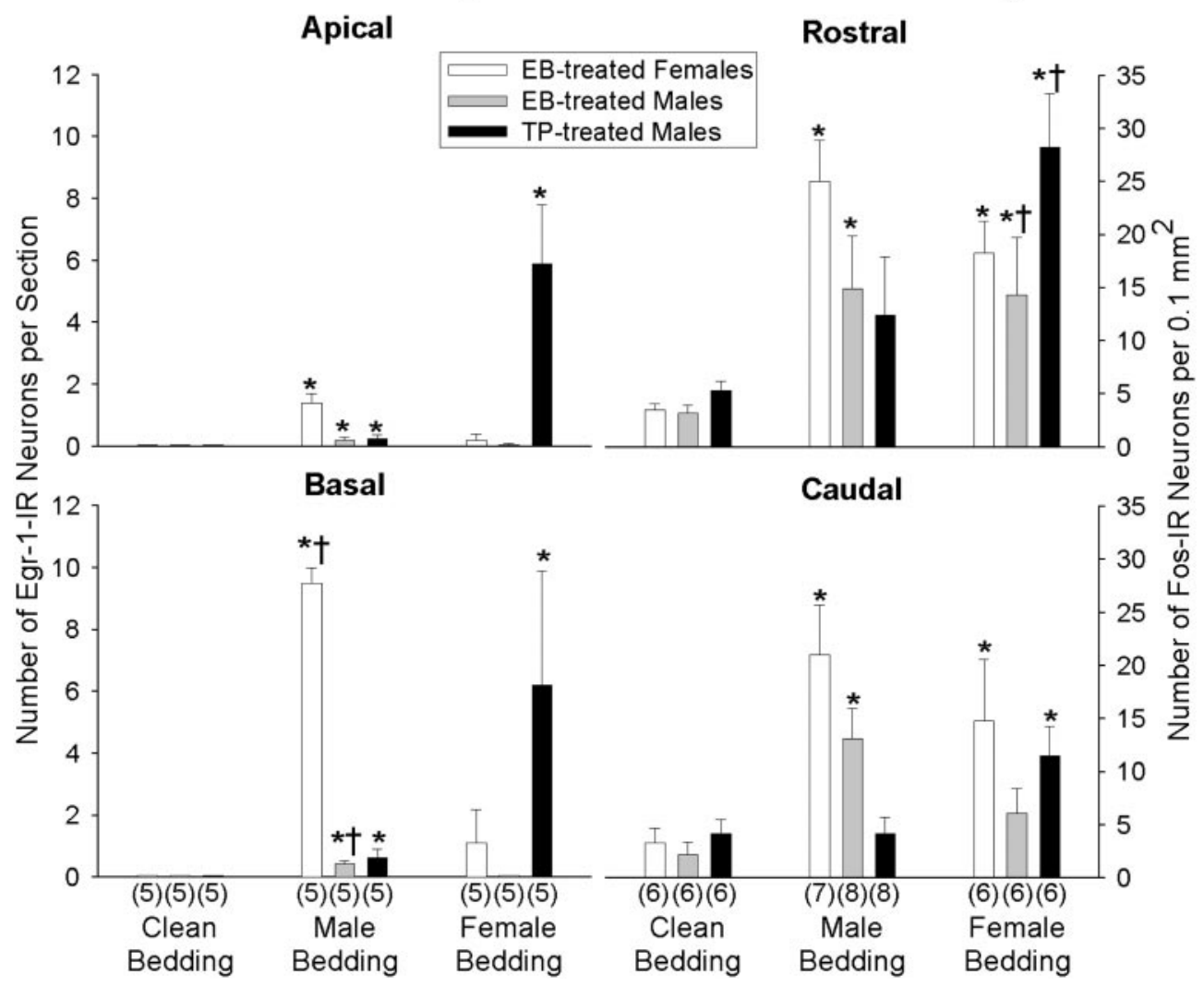

AOB - Mitral Cell Layer

Rostral

Figure 2. The mean \pm SEM numbers of IEG-IR neurons induced in the VNO and AOB are shown for gonadectomized subjects treated with EB or TP after exposure to clean bedding, soiled male bedding, or soiled female bedding. Data are presented for Egr-1-IR neurons in the apical and basal VNO neuroepithelium and Fos-IR neurons in the rostral and caudal mitral cell layer of the AOB. The number of subjects in each group is given in parentheses. Asterisks in each graph denote a significant $(p<0.05)$ effect of soiled versus clean bedding (Kruskal-Wallis ANOVA with Mann-Whitney $U$ Tests). Daggers represent significant differences between rostral and caudal or apical and basal means for a particular group, as determined by two-way ANOVAs, followed by Student-Newman-Keuls post hoc tests.
Table 1. Number of Fos-IR neurons in the granule cell layer of the AOB

\begin{tabular}{lcll} 
& Clean bedding & Male bedding & Female bedding \\
\hline Rostral & & & \\
EB females & $8 \pm 2(6)$ & $67 \pm 19(6)^{*}$ & $52 \pm 14(6)^{*}$ \\
EB males & $12 \pm 2(7)$ & $31 \pm 8(8)^{*}$ & $35 \pm 12(8)$ \\
TP males & $16 \pm 4(6)$ & $23 \pm 13(6)$ & $94 \pm 28(6)^{*} \dagger$ \\
Caudal & & & \\
EB females & $3 \pm 1(6)$ & $50 \pm 12(6)^{*}$ & $29 \pm 12(6)^{*}$ \\
EB males & $4 \pm 1(7)$ & $22 \pm 7(8)^{*}$ & $15 \pm 4(8)^{*}$ \\
TP males & $4 \pm 1(6)$ & $11 \pm 6(6)$ & $35 \pm 14(6)^{*}$
\end{tabular}

The mean \pm SEM number of Fos-IR neurons in the granule cell layer of the AOB are compared in subjects treated with EB or TP and exposed to clean bedding, soiled male bedding, or soiled female bedding. The number of subjects in each group is given in parentheses. Asterisks denote a significant $(p<0.05)$ effect of soiled versus clean bedding (Kruskal-Wallis ANOVA with post hoc Mann-Whitney $U$ tests). Daggers indicate significant differences between rostral and caudal means for a particular group, as determined by two-way ANOVAs, followed by StudentNewman-Keuls post hoc tests.

caudal AOB of TP- and EB-males (Fig. 2). In the granule cell layer, there was a significant effect of location $\left(F_{(1,39)}=26.5 ; p<\right.$ $0.01)$ and a significant treatment $\times$ location interaction $\left(F_{(2,39)}=\right.$ 4.2; $p<0.03)$. Post hoc comparisons indicated that these effects were attributable primarily to the greater response of TP- than EB-males to female pheromones in the rostral portion of the granule cell layer (Table 1).

\section{Maps of neuronal activation by pheromones}

Patterns of neuronal activation within the two zones of the VNO and AOB are depicted in composite maps of IEG activation that show group responses to different pheromones (Fig. 3). (Control groups are not shown because induction of IEG-IR by clean bedding was minimal.) These maps reveal that there is a widespread overall distribution of neuronal activation within the $\mathrm{VNO}$ and AOB, and furthermore, they reflect the discordance between patterns of neuronal activation within the zones of each structure. For example, heavy basal neuron activation in the $\mathrm{VNO}$ of EB-females exposed to male bedding did not correspond with preferential IEG induction in the caudal region of the AOB. Similarly, in EB-males, exposure to female pheromones resulted in significant IEG induction in the AOB but virtually no response in the VNO.

Despite finding zonal differences in the response of VNO neurons, Fos-IR neurons were not induced differentially within specific rostral and caudal subregions of the AOB by different pheromones. To test the possibility that other patterns of IEG activation may have been induced in the AOB, we examined whether Fos activation across individual grid squares differed among groups. In the granule cell layer of EB-males and EBfemales, a statistically significant bedding type $\times$ sex of subject $\times$ grid interaction was found $\left(F_{(1,71)}=1.29 ; p=0.05\right)$, suggesting that a different distribution and/or number of Fos-IR neurons was observed across grid squares in EB-males and EB-females in response to different pheromones. This result is clearly represented in the spatial maps of these groups, which illustrate that IEG activation in granule cells of females exposed to male bedding is greater and more widespread than in EB-males or EB-females exposed to female bedding (Fig. 3). In contrast, the bedding type $\times$ sex of subject $\times$ grid interaction was not significant for AOB mitral cells, indicating that specific patterns of IEG activation in this region did not differ among groups.

Similarly, in EB- and TP-males exposed to male and female pheromones, the interaction of bedding type $\times$ hormone of 


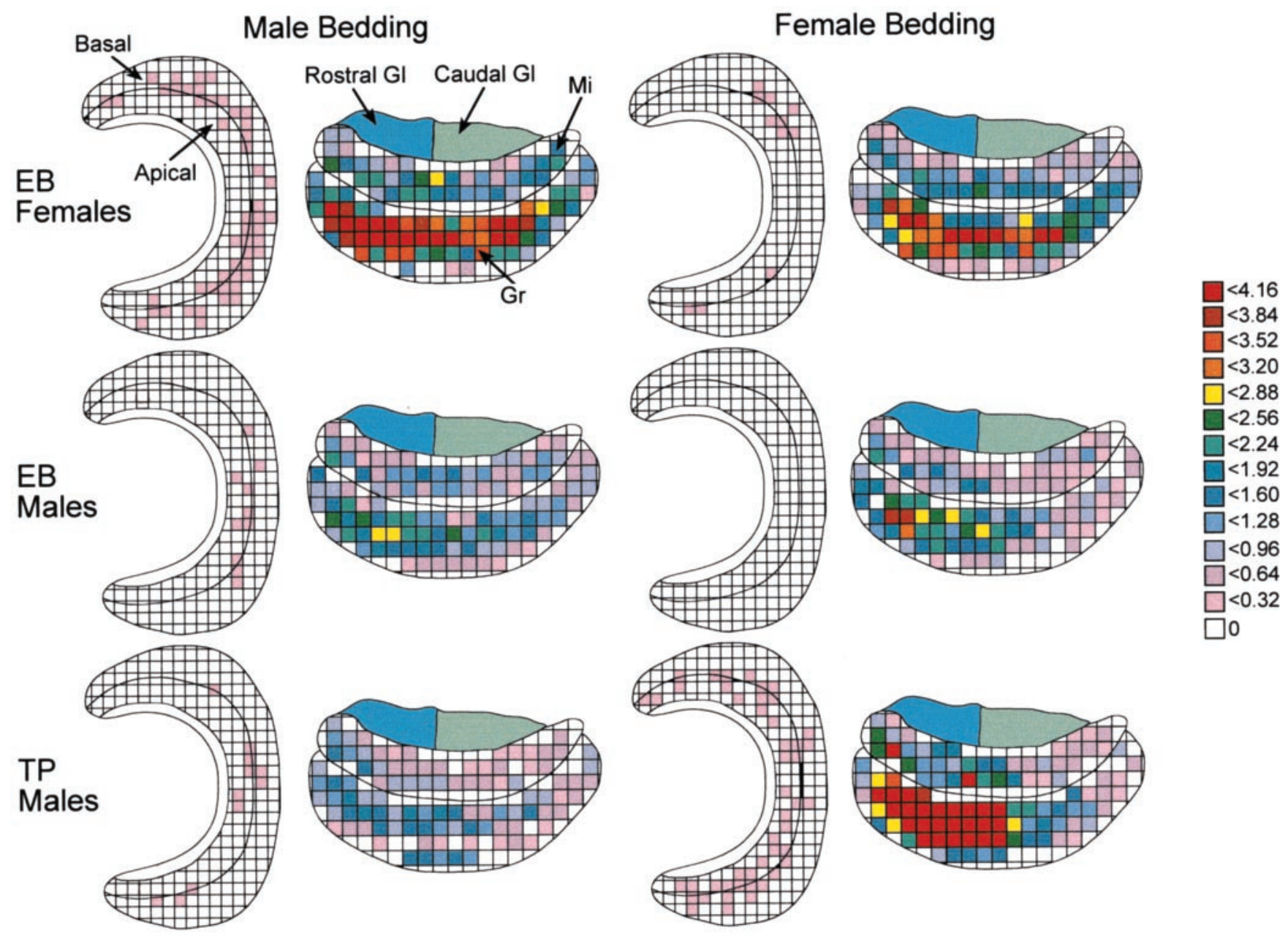

Figure 3. Composite spatial maps illustrate group responses to pheromones in the VNO and AOB. Grid squares were assigned a color based on the mean number of IEG-immunoreactive neurons (Egr-1 in the VNO; Fos in the AOB) induced in a particular square from all subjects in that group. $n=$ 6 for EB-female and TP-treated male groups; $n=8$ for both groups of EB-males. Gl, Glomerular layer; $M i$, mitral cell layer; $G r$, granule cell layer.

subject $\times$ grid indicated that the distribution of Fos-IR across grids in AOB granule cells differed significantly between groups $\left(F_{(1,71)}=4.53 ; p<0.05\right)$. Consistent with this statistical result, the composite map of TP-males exposed to female bedding stands out from the other groups with respect to the pattern and level of IEG response. In contrast, patterns of Fos distribution in AOB mitral cells were not significantly different among groups (Fig. 3).

Overall, comparison of patterns of Fos distribution in the AOB granule cell layer confirmed our general finding that the level of Fos activation depended on the sex and hormonal status of the subject, as well as the pheromone to which subjects were exposed. Conversely, statistical comparison of the composite figures failed to show that any of these factors influenced the distribution of Fos activation in the AOB mitral cell layer.

\section{DISCUSSION}

\section{Regional responses to male and female pheromones in the VNO and AOB}

Our results provide evidence that neurons within defined subdivisions of the VNO respond differentially to pheromones. Moreover, responses to the same pheromones differed dramatically in the VNO of males and females, and these responses in males depended on circulating steroids. In particular, pheromonal activation of neurons in the VNO was greatest in animals treated with hormones normally produced during conditions of breeding and was selective for pheromones of the opposite sex. The total number of Egr-1-IR neurons in the VNO was far greater in females than in either group of males after exposure to soiled male bedding. Similarly, exposure to soiled female bedding induced greater Egr-1-IR in the VNO of TP-males compared with EB-treated males and females.

Notably, pheromones induced different levels of activation in VNO zones of males and females. More basal than apical neurons were activated in EB-treated animals after exposure to soiled female than male bedding. This result suggests that receptors for male pheromones may be expressed primarily in basal neurons. Conversely, there were no apical-basal differences in the response of either sex to female pheromones. These observations suggest that, although some overlap exists, receptors for male and female pheromones may occur preferentially in different layers of the VNO neuroepithelium.

In the AOB, evidence for zonal differences in IEG induction by pheromones was less clear. A widespread dispersion of granule cell responses and an even wider set of mitral cell responses were found after exposure to male and female pheromones. Even in the male groups exposed to female bedding, which were the only groups with significant rostral-caudal differences, the greatest numbers of Fos-IR neurons were distributed across the rostro- 
caudal boundary of the AOB. We did detect statistically significant group differences in the overall distribution of Fos-IR across individual grid squares in AOB granule cells, but inspection of the composite maps indicates that any differences between groups could not be attributed to isolated areas of neuronal activity.

These results were contrary to the expectation that a correspondence would be found between IEG induction in the VNO and $\mathrm{AOB}$, especially in light of evidence that the segregation found in VNO projections to AOB glomeruli extends to the population of AOB mitral cells; anteriorly located mitral cells send primary dendrites to rostral AOB glomeruli, and caudal mitral cell dendrites extend to the caudal glomerular division (Jia and Halpern, 1997; von Campenhausen et al., 1997). One possibility is that, in some cases, subtle gradients of rostral-caudal electrical activity may actually have been induced in mitral cells after exposure to soiled bedding that did not result in IEG expression in these cells. However, AOB granule cells, other mitral cells, and/or centrifugal afferents all may provide inputs to mitral cells. Perhaps integration of these inputs by individual mitral cells results in an overall response that does not correspond precisely to the activity of apical and basal VNO neurons. Electrical recording of mitral cell responses in the rostral versus caudal AOB to different stimuli will be necessary to help resolve this issue.

It was also curious that, for some groups, such as EB-treated males and females exposed to female bedding, stimulation that produced a minimal IEG response in the VNO was accompanied by substantial IEG induction in the AOB. It could be that certain classes of pheromones do not induce IEGs in VNO neurons or induce IEGs other than those we studied. The relationship between mitral cell firing, the induction of different IEGs, and the degree to which rostral and caudal boundaries are functionally maintained in the deeper cell layers of the AOB remains unknown.

In female mice, urine-derived compounds applied to VNO slices resulted in a transient $\mathrm{Ca}^{2+}$ rise in a small number of neurons located in the apical portion of the neuroepithelium (Leinders-Zufall et al., 2000). In addition, patch-clamp recordings in VNO slices have demonstrated that apical neurons from female Wistar rats responded best to male urine from Wistar rats, whereas basal neurons responded to male urine from mice, hamsters, or rats of another strain (Inamura et al., 1999). These studies suggest that conspecific urinary pheromones from males activate apical neurons in the VNO of females, which contrast with our observations that basal VNO neurons in females respond preferentially to pheromones present in soiled male bedding. Several explanations for this discrepancy are possible. First, in the absence of independent labels to identify the two zones, it is not clear from VNO slice studies whether only apical neurons were being examined. Second, these studies used tissue obtained from gonadally intact subjects, so the steroid environment was not rigorously controlled. As our present study indicates, circulating hormones can dramatically affect the IEG response of VNO neurons to pheromones. Finally, our study used soiled bedding, a natural stimulus composed of a mixture of pheromonal compounds. Some of these compounds from male bedding could preferentially activate receptors in apical VNO neurons of females. Indeed, there were some Egr-1-IR (and Fos-IR) neurons in the apical zone that were activated in the present study.

To understand how pheromones are coded by the accessory olfactory system, it will be useful to identify neurons in the VNO and $\mathrm{AOB}$ that respond to individual pheromones. Unfortunately, few compounds suspected to be pheromones have been identified in mice, and of these, the best characterized have been isolated from males (Jemiolo et al., 1986). Although certain of these molecules when presented to mice have been shown to be behaviorally and physiologically active (Jemiolo et al., 1986; LeindersZufall et al., 2000), in urine they occur bound to major urinary proteins (MUPs). In fact, in female mice presented with the urine-derived compounds 2,3-dehydro-exo-brevicomin and 2-secbutyl-4,5-dihydrothiazole, induction of c-fos mRNA in the AOB occurred only when the compounds were presented along with MUPs (Guo et al., 1997). Conversely, when a combination of 2,3-dehydro-exo-brevicomin and 2-sec-butyl-4,5-dihydrothiazole was applied to the nares of female mice, Egr-1 was selectively induced in clusters of mitral cells along the margins of the caudal AOB (Brennan et al., 1999). Additional work will be required to resolve these issues and to determine whether different constituents of soiled bedding can induce IEGs in specific neurons of the VNO.

\section{Sex dimorphism and activational effects of sex steroids in VNO and AOB}

Sex differences in the VNO response to male pheromones could reflect differences in the distribution of VNO receptor subtypes (Herrada and Dulac, 1997) or be related to sex dimorphisms in VNO size and/or number of neurons (Segovia and Guillamon, 1982). Alternatively, as discussed below, sex differences in steroid-sensitive centrifugal inputs may mediate VNO responses to pheromones.

The sex dimorphism and activational effects of steroid hormones on neuronal responsiveness to different pheromones in the VNO were not as evident at the level of the AOB. It is possible that signals arriving from the VNO are modulated by interneurons of the AOB or by centrifugal inputs. For example, noradrenergic projections from the locus ceruleus, which have been shown to facilitate the formation of olfactory memory in mice, could influence the response properties of AOB neurons to signals transmitted from the VNO (Rosser and Keverne, 1985; McLean et al., 1989).

The sexually dimorphic response of basal VNO neurons in this study confirm and extend results of a previous study (Halem et al., 1999) in which estrogen-primed females responded to soiled male bedding with a greater induction of Fos-IR neurons in the VNO than did oil-treated females. Because there are no data indicating that steroid hormone receptors are localized in the VNO, it seems likely that the effects of steroids on VNO responsiveness to pheromones occur indirectly. One possibility is that the VNO receives modulatory inputs from steroid-sensitive neurons elsewhere in the nervous system. Recently, it has been proposed that epinephrine may act in the olfactory epithelium to modulate the firing pattern of olfactory receptor neurons in response to an odor (Firestein and Menini, 1999; Kawai et al., 1999). Although yet to be confirmed in vivo, these results suggest that centrifugal noradrenergic afferents, probably originating in the superior cervical ganglia (SCG), may alter the responsiveness of olfactory neurons under particular environmental or hormonal conditions (Firestein and Menini, 1999).

Similarly, we suggest that sex steroids may act indirectly on noradrenergic inputs to the VNO to alter the sensitivity of neurons to stimulation by particular pheromones. It is known that norepinephrine is present in the mouse VNO (Zancanaro et al., 1997) and that the VNO is innervated by noradrenergic fibers (Meredith and O'Connell, 1979). These noradrenergic inputs 
probably originate in the SCG (Meredith and O'Connell, 1979), which is larger in male than female rats and is sensitive to activation by testosterone (Dibner and Black, 1978; Wright and Smolen, 1983). In this manner, noradrenergic inputs could directly modulate the firing rate of VNO neurons activated by specific pheromones. Alternatively, noradrenergic fibers innervating the VNO could indirectly affect pheromonal responsiveness by regulating activity of the VNO pump that draws pheromones into the VNO lumen (Meredith, 1994) or by controlling the release of pheromone binding proteins from glands associated with the VNO (Khew-Goodall et al., 1991; Miyawaki et al., 1994).

Our results provide in vivo evidence of a sex dimorphism and steroidal modulation of the responsiveness of primary VNO receptors to pheromones. In addition, a recent report that used a multi-electrode array to record from sheets of VNO neuroepithelia found that there are neurons in male and female VNOs that respond uniquely to pheromones of one sex (Holy et al., 2000). In other sensory modalities as well, such as the auditory system of the frog (Wilczynski, 1986; Ryan et al., 1990), functional differences in the primary sensory organs of males and females have been described. In such systems, sex differences in perception begin at the sensory receptor. This design, which does not require downstream processing to distinguish between different sensory cues, may ensure the accurate perception of stimuli that are relied on for such critical functions as mate recognition and reproductive behavior.

\section{REFERENCES}

Bakker J, Baum MJ, Slob AK (1996) Neonatal inhibition of brain estrogen synthesis alters adult neural fos responses to mating and pheromonal stimulation in the male rat. Neuroscience 74:251-260.

Belluscio L, Koentges G, Axel R, Dulac C (1999) A map of pheromone receptor activation in the mammalian brain. Cell 97:209-220.

Brennan PA, Schellinck HM, Keverne EB (1999) Patterns of expression of the immediate-early gene egr-1 in the accessory olfactory bulb of female mice exposed to pheromonal constituents of male urine. Neuroscience 90:1463-1470.

Bruce HM (1959) An exteroreceptive block to pregnancy in the mouse. Nature 84:105.

Cherry JA, Davis RL (1995) A mouse homolog of dunce, a gene important for learning and memory in Drosophila, is preferentially expressed in olfactory receptor neurons. J Neurobiol 28:102-113.

Coquelin A, Clancy AN, Macrides F, Noble EP, Gorski RA (1984) Pheromonally induced release of luteinizing hormone in male mice: involvement of the vomeronasal system. J Neurosci 4:2230-2236.

Dibner MD, Black IB (1978) Biochemical and morphological effects of testosterone treatment on developing sympathetic neurons. J Neurochem 30:1479-1483.

Dulac C, Axel R (1995) A novel family of genes encoding putative pheromone receptors in mammals. Cell 83:195-206.

Firestein S, Menini A (1999) The smell of adrenaline. Nat Neurosci 2:106-108.

Guillot PV, Chapouthier G (1996) Olfaction, GABAergic neurotransmission in the olfactory bulb, and intermale aggression in mice: modulation by steroids. Behav Genet 26:497-504.

Guo J, Zhou A, Moss RL (1997) Urine and urine-derived compounds induce c-fos mRNA expression in accessory olfactory bulb. NeuroReport 8:1679-1683.

Halem HA, Cherry JA, Baum MJ (1999) Vomeronasal neuroepithelium and forebrain fos responses to male pheromones in male and female mice. J Neurobiol 39:249-263.

Herrada G, Dulac C (1997) Novel family of putative pheromone receptors in mammals with a topographically organized and a sexually dimorphic distribution. Cell 90:763-773.

Holy TE, Dulac D, Meister M (2000) Responses of vomeronasal neurons to natural stimuli. Science 289:1569-1572.

Inamura K, Kashiwayanagi M, Kurihara K (1999) Regionalization of
Fos immunostaining in rat accessory olfactory bulb when the vomeronasal organ was exposed to urine. Eur J Neurosci 11:2254-2260.

Jemiolo B, Harvey S, Novotny M (1986) Promotion of the Whitten effect in female mice by synthetic analogs of male urinary constituents. Proc Natl Acad Sci USA 83:4576-4579.

Jia C, Halpern M (1996) Subclasses of vomeronasal receptor neurons differential expressions of $G$ proteins $\left(G_{i \alpha 2}\right.$ and $\left.G_{o \alpha}\right)$ and segregated projections to the accessory olfactory bulb. Brain Res 719:117-128.

Jia C, Halpern M (1997) Segregated populations of mitral/tufted cells in the accessory olfactory bulb. NeuroReport 8:1887-1890.

Johnston RE, Bronson FH (1982) Endocrine control of female mouse odors that elicit luteinizing hormone surges and attraction in males. Biol Reprod 27:1174-1180.

Kawai F, Kurahashi T, Kaneko A (1999) Adrenaline enhances odorant contrast by modulating signal encoding in olfactory receptor cells. Nat Neurosci 2:133-138.

Kelliher KR, Chang YM, Wersinger SR, Baum MJ (1998) Sex difference and testosterone modulation of pheromone-induced neuronal Fos in the ferret's main olfactory bulb and hypothalamus. Biol Reprod 59:1454-1463.

Khew-Goodall Y, Grillo M, Getchell ML, Danho W, Getchell TV, Margolis FL (1991) Vomeromodulin, a putative pheromone transporter: cloning, characterization, and cellular localization of a novel glycoprotein of lateral nasal gland. FASEB J 5:2976-2982.

Lau YE, Cherry JA (2000) Distribution of PDE4A and $\mathrm{G}_{\mathrm{o} \alpha}$ immunoreactivity in the accessory olfactory system of the mouse. NeuroReport 11:27-32.

Leinders-Zufall T, Lane AP, Puche AC, Ma W, Novotony MV, Shipley MT, Zufall F (2000) Ultrasensitive pheromone detection by mammalian vomeronasal neurons. Nature 405:792-796.

Lombardi JR, Vandenbergh JG (1977) Pheromonally induced sexual maturation in females: regulation by the social environment of the male. Science 196:545-546.

Matsunami H, Buck LB (1997) A multigene family encoding a diverse array of putative pheromone receptors in mammals. Cell 90:775-784.

McLean JH, Shipley MT, Nickell WT, Astion-Jones G, Reyher CKH (1989) Chemoanatomical organization of the noradrenergic input from locus coeruleus to the olfactory bulb of the adult rat. J Comp Neurol 285:339-349.

Meredith M (1994) Chronic recording of vomeronasal pump activation in awake behaving hamsters. Physiol Behav 56:345-354

Meredith M, O'Connell J (1979) Efferent control of stimulus access to the hamster vomeronasal organ. J Physiol (Lond) 286:301-316.

Miyawaki A, Matsushita F, Ryo Y, Mikoshiba K (1994) Possible pheromone-carrier function of two lipocalin proteins in the vomeronasal organ. EMBO J 13:5835-5842.

Nyby J, Wysocki CJ, Whitney G, Dizinno G, Schneider J (1979) Elicitation of male mouse (Mus musculus) ultrasonic vocalizations. I. Urinary cues. J Comp Physiol Psychol 93:957-975.

Rodriguez I, Feinstein P, Mombaerts P (1999) Variable patterns of axonal projections of sensory neurons in the mouse vomeronasal system. Cell 97:199-208.

Rosser AE, Keverne EB (1985) The importance of central noradrenergic neurons in the formation of an olfactory memory in the prevention of pregnancy block. Neuroscience 15:1141-1147.

Ryan MJ, Fox JH, Wilczynski W, Rand AS (1990) Sexual selection for sensory exploitation in the frog Physalaemus pustulosus. Nature 343:66-67.

Ryba N, Tirindelli R (1997) A new multigene family of putative pheromone receptors. Neuron 19:371-379.

Segovia S, Guillamon A (1982) Effects of sex steroids on the development of the vomeronasal organ in the rat. Dev Brain Res 5:209-212.

van der Lee S, Boot LM (1959) Spontaneous pseudopregnancy in mice. Acta Physiol Pharmacol Nederland 4:442-444.

von Campenhausen H, Yoshihiro Y, Mori K (1997) OCAM reveals segregated mitral/tufted cell pathways in developing accessory olfactory bulb. NeuroReport 8:2607-2612.

Whitten WK (1959) Occurrence of anoestrus in mice caged groups. J Endocrinol 18:102-107.

Wilczynski W (1986) Sexual differences in neural tuning and their effect on active space. Brain Behav Evol 28:83-94.

Wright LL, Smolen AJ (1983) Neonatal testosterone treatment increases neuron and synapse numbers in male rat superior cervical ganglion. Dev Brain Res 8:145-153.

Zancanaro C, Mucignat C, Bolner A, Sbarbatl A, Nordera GP, Osculati F (1997) Biogenic amines in the vomeronasal organ. Chem Senses 22:439-445. 